\title{
Spontaneous resolution of iatrogenic dissection of the left main coronary artery extending to the thoracic and abdominal aorta
}

\author{
Bartłomiej Perek ${ }^{1}$, Maciej Lesiak², Marek Jemielity ${ }^{1}$ \\ 1Department of Cardiac Surgery and Transplantology, Poznan University of Medical Sciences, Poland \\ 2Department of Cardiology, Poznan University of Medical Sciences, Poland
}

Postep Kardiol Inter 2013; 9, 2 (32): 194-197

DOI: $10.5114 /$ pwki.2013.35460

\begin{abstract}
latrogenic coronary artery dissection extending to the ascending aorta is a rare complication of percutaneous coronary interventions. Coronary stent implantation is usually sufficient to control the injury. In this report we describe an unusual case of spontaneous resolution of both left main coronary artery and aortic wall dissection. The patient was not operated on due to the location of the initial tear in the distal part of the left main coronary artery. Moreover, in computed tomography (CT) thrombus formation in the false lumen of the dissected aorta was seen. The in-hospital course was uneventful. The last follow-up CT showed complete resolution of dissection.
\end{abstract}

Key words: coronary angiography, complications, aortic dissection.

\section{Introduction}

latrogenic coronary artery dissection extending proximally to the aortic root and then further aortic segments is a rare but potentially fatal complication of percutaneous coronary interventions ( $\mathrm{PCls}$ ) [1]. Usually it may be treated successfully with coronary stent implantation. If stenting fails or if aortic wall dissection is very extensive, urgent cardiac surgical operation is recommended.

In this report we describe an unusual case of a woman with iatrogenic left main coronary artery and aortic dissection who was treated medically with complete spontaneous resolution of the disease.

\section{Case report}

A 63-year old woman was admitted to the Cardiac Surgical Department directly from the catheterization laboratory of the regional hospital with the diagnosis of iatrogenic left main coronary and aortic wall dissection. Seven months earlier she was treated in the cardiology department due to acute coronary syndrome (ST-segment elevation myocardial infarction - STEMI) and underwent uneventful angioplasty of the proximal segment of the cir- cumflex artery with bare metal stent (BMS) implantation. After the procedure the patient was discharged home and scheduled for elective angioplasty of the left anterior descending artery (LAD). After successful stent deployment in the proximal segment of the $L A D$, a dissection in the distal left main coronary artery was noticed (Figure 1). The dissection neither reduced significantly the lumen of the left main trunk nor compromised the blood flow. Unfortunately, it extended proximally to the ascending aortic wall. Since the guide-wire had already been pulled out, the operator decided to stop the procedure and refer the patient immediately to the Cardiac Surgical Department. On admission to this department the patient was asymptomatic (no pain, no symptoms of either heart failure or any organ malperfusion; arterial pressure $110 / 70 \mathrm{~mm} \mathrm{Hg}$ with no difference between left and right forearm). She immediately underwent computed tomography angiography (CTA) that confirmed dissection of the ascending aorta, aortic arch and the descending aorta down to the middle part of the abdominal aorta. The aortic wall was slightly dilated and its external diameter did not exceed $45 \mathrm{~mm}$ in any segment (42 $\mathrm{mm}$ in the ascending aorta, 35 in the descending thoracic and $29 \mathrm{~mm}$ in the abdominal aorta) (Figures

\section{Corresponding author:}

Bartłomiej Perek MD, Department of Cardiac Surgery and Transplantology, Poznan University of Medical Sciences, 1/2 Długa St, 61-848 Poznan, Poland, tel.: +48 6185492 10, fax: +48 6185490 85, e-mail: bperek@yahoo.com

Received: 25.03.2013, accepted: 10.05.2013. 
$2 \mathrm{~A}$ and $2 \mathrm{~B}$ ). Additionally, in this examination a distal portion of the left main stem was diagnosed as the most likely site of the initial tear of the vessel wall. Moreover, thrombus partially filling the false channel in the ascending aorta, aortic arch and thoracic segment of the descending aorta was visualized. In transthoracic echocardiography, no abnormalities with respect to the cardiac chamber dimensions or cardiac valve function (particularly the aortic valve) were noted. No pericardial effusion was found. After detailed analysis of the clinical status (no symptoms), the mechanism of dissection (iatrogenic injury), the location of the initial tear of the vessel wall (the distal segment of the left main stem) and other CTA findings (thrombus in the false channel), the cardiac surgeon decided not to operate on the patient and to treat her medically with strict control of arterial pressure and heart rate. The patient was subjected to daily echocardiographic observation and the further in-hospital stay was uneventful; therefore 2 weeks later she was discharged and referred to the rehabilitation center. In the following months she underwent systematic clinical and CTA outpatient follow-up. The last CTA (36 months after the unfortunate $\mathrm{PCI}$ ) revealed a complete occlusion of the false lumen of the aorta (Figure 3). Maximal external diameter of the ascending aorta was $43 \mathrm{~mm}$, aortic arch $36 \mathrm{~mm}$ and the descending aorta $31 \mathrm{~mm}$.

\section{Discussion}

The incidence of iatrogenic coronary artery dissection (predominantly right) during a $\mathrm{PCl}$ procedure is less than $1 \%$. Usually it is limited to the coronary artery itself and is usually controlled by stent implantation [2]. Iatrogenic aortic wall dissection following coronary artery injury during
$\mathrm{PCl}$ is even more uncommon and accounts for approximately $0.02 \%$ of all $\mathrm{PCl}$ cases [1]. However, this complication may be underreported; hence the actual number is not known. Consequently, an optimal therapeutic strategy of iatrogenic aortic dissection has not been elaborated and remains controversial. Our current knowledge is largely based on case reports [3-5], so the choice of treatment strategy is often based on the experience and clinical intu-

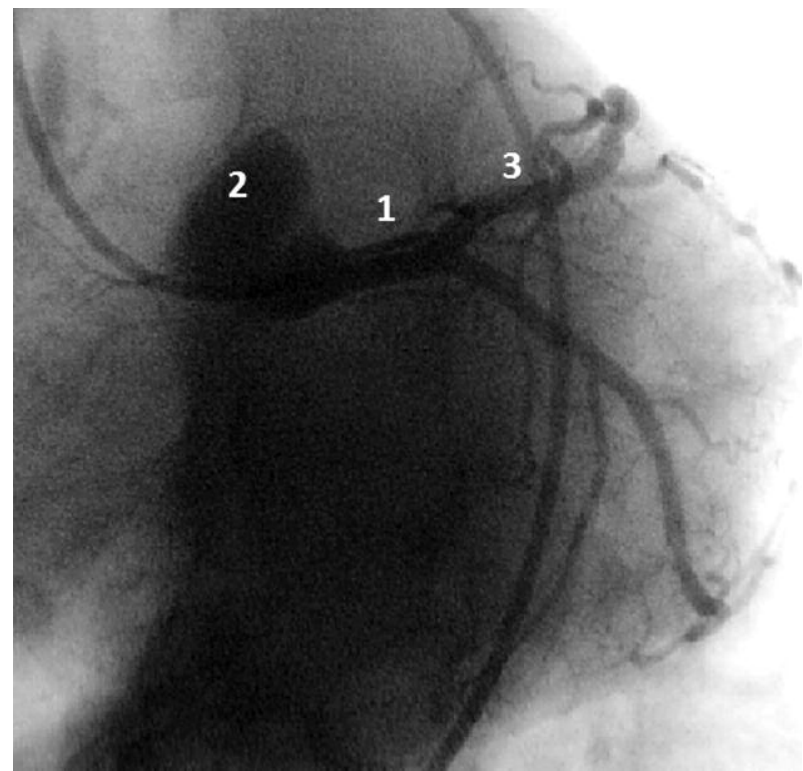

Fig. 1. Left main dissection (1) extending to the ascending aorta (2) was visualized in coronary angiography after successful angioplasty of the left anterior descending artery (3). Left main dissection did not compromise blood flow
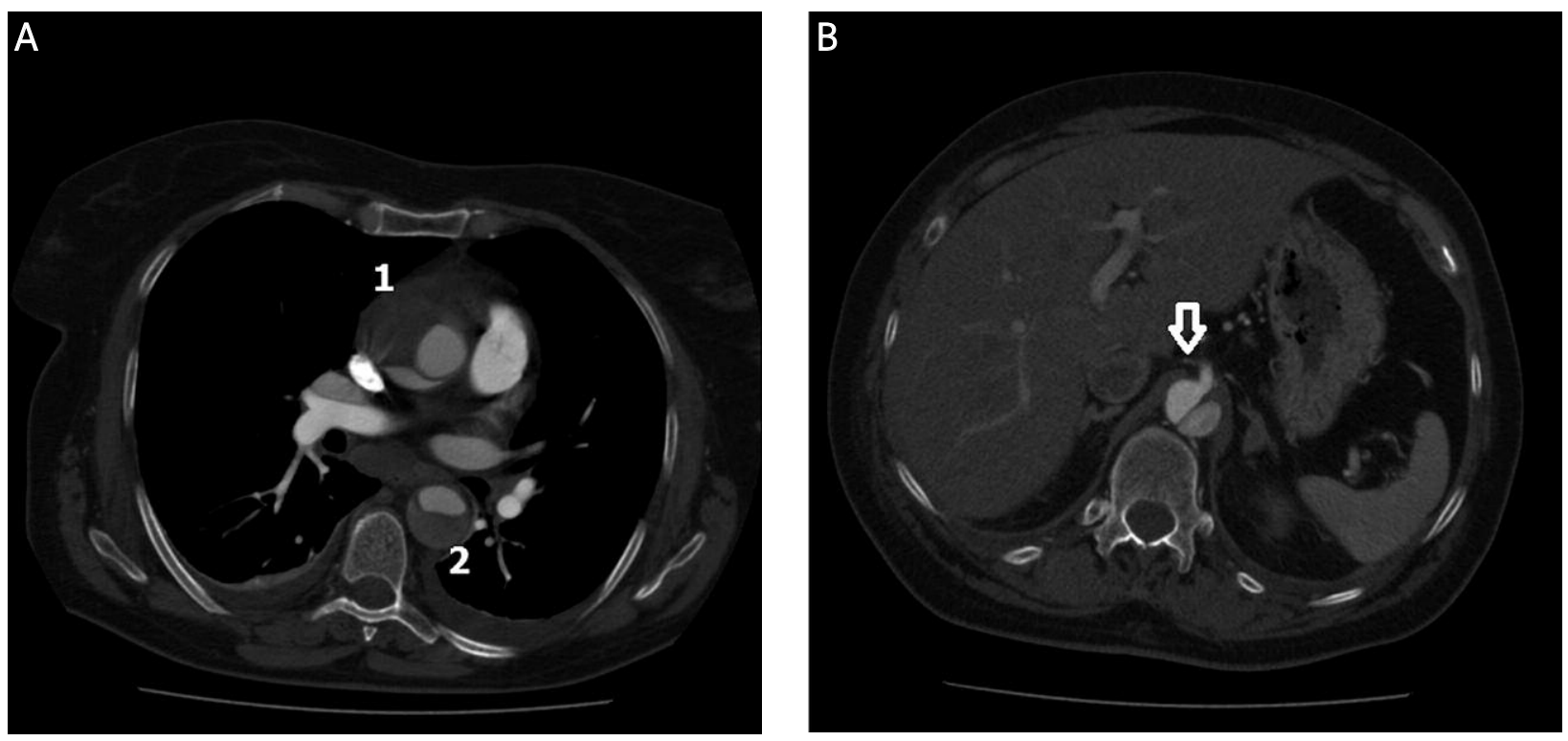

Fig. 2. A - A CT scan of the chest. Ascending aorta (1) is dissected with thrombus partially filling false aneurysm. In the descending thoracic aorta (2) thrombus is filling the whole false lumen. B - A CT scan of the abdomen. Abdominal aorta at the level of the celiac trunk $(\downarrow)$ is dissected while maintaining flow through both true and false lumen 

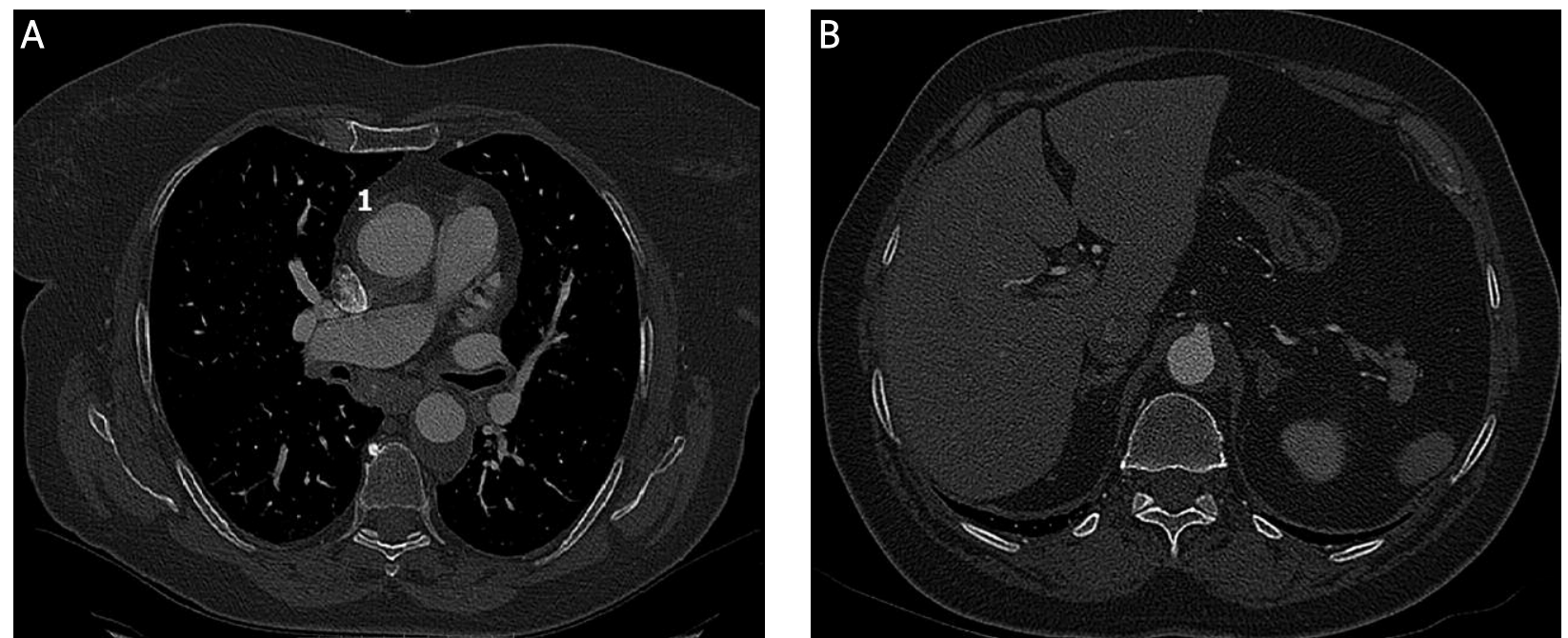

Fig. 3. 36-month follow-up CT scan with complete resolution of the dissection in both the thoracic (A) and abdominal (B) segment. True lumen of the aorta, but not the external diameter, especially its ascending part (1), is markedly enlarged after 36 months as compared with the image obtained just after iatrogenic injury

ition. Each patient should be treated individually and all factors that may potentially impact the natural course of the disease require careful consideration.

Most iatrogenic aortic dissections beginning at the proximal segments of coronary arteries can be managed with immediate coronary stenting sealing the entry site, and should be followed by close observation and medical therapy controlling the blood pressure and the heart rate $[1,4,6]$. In our case, the interventional cardiologist in the regional hospital decided not to implant the stent due to the unfavorable tear location and preserved blood flow through the left main stem. Some authors claim that surgical intervention should be preferred if the dissection extends more than $4 \mathrm{~cm}$ up the aorta [1]. However, even when the dissection is extensive, in some subjects stenting of the coronary ostium may be enough to control the dissection progression $[4,5]$. Rapid progression of aortic dissection with the involvement of the arch vessels and other complications, such as aortic regurgitation, malperfusion of the peripheral organs and pericardial effusion or tamponade, mandate surgical intervention. In our case, an inconvenient location of the entry tear as well as the lack of any aforementioned complications prompted us to initiate medical therapy. Our management in this particular case seems to be justified by the late outcome. We should stress that the most crucial step in a surgical repair of the dissected aorta or any other vessel is the replacement of an arterial segment containing the culprit tear. A direct surgical access to the distal portion of the trunk of the left coronary artery is more than demanding. Moreover, proximal ascending aortic wall dissection is usually accompanied by massive hematoma of the adjacent tissue involving the right ventricular outflow tract. This location makes surgical repair of the left main stem practically impossible.
According to the current guidelines, all patients diagnosed with ascending aortic dissection (type A) should be urgently evaluated for emergent surgical repair because of the high risk of life-threatening complications (class I recommendation) [7]. Risk of death among patients treated medically is very high, particularly within the first $48 \mathrm{~h}$ from symptom onset (1\% every $1 \mathrm{~h}$ ) [8]. The International Registry of Acute Aortic Dissection (IRAD) showed significantly higher mortality among patients with type $A$ dissection who did not receive surgery (58\%) as compared to those treated surgically (26\%) [9]. However, the aforementioned guidelines are based on clinical data of all individuals with acute aortic dissection irrespective of the initial cause. Dissections were predominantly spontaneous, whereas iatrogenic ones accounted for less than $5 \%$ of them [9].

In our opinion, the mechanism of aortic dissection is of paramount importance regarding therapeutic management. Spontaneous aortic wall dissection appears often as an extensive tear involving uncommonly the whole aortic circumference. Additionally, aortic wall obtained intraoperatively for histological study showed degenerative changes that confined particularly the elastic fibers, one of the critical components of the wall matrix determining its mechanical and functional properties [10]. In such cases that account for the majority of subjects treated for acute aortic dissection, spontaneous resolution is probably impossible, so conservative medical management must not be recommended. Another issue is iatrogenic dissection due to mechanical reasons such as guiding catheters, wires, balloons or stents that cause usually small tears, a few millimeters in length. Thus, in these particular, but rare cases the chances for spontaneous wall healing seem to be relatively high. 


\section{Conclusions}

In selected cases iatrogenic left main dissection extending to the aortic wall may be treated medically, as long as the flow in the left main stem is not compromised. However, each patient requires an individual meticulous approach taking into account the clinical appearance and the findings from all available imaging modalities. Careful echocardiographic follow-up for pericardial effusion and CT scanning for aorta assessment are mandatory after iatrogenic aortic dissection.

\section{References}

1. Dunning DW, Kahn JK, Hawkins ET, et al. latrogenic coronary artery dissections extending into and involving the aortic root. Catheter Cardiovasc Interv 2000; 51: 387-393.

2. Zidi M, Nallet O, Esteve JB, et al. Extensive iatrogenic coronary dissection during coronary angioplasty: a series of 19 consecutive patients. Ann Cardiol Angeiol (Paris) 2010; 59: 306-310.

3. Yip HK, Wu CJ, Yeh KH, et al. Unusual complication of retrograde dissection to the coronary sinus of valsalva during percutaneous revascularization: a single-center experience and literature review. Chest 2001; 119: 493-501.

4. Carstensen $S$, Ward MR. latrogenic aortocoronary dissection: the case for immediate aortoostial stenting. Heart Lung Circ 2008; 17: 325-329.

5. Park IW, Min PK, Cho DK, et al. Successful endovascular treatment of iatrogenic coronary artery dissection extending into the entire ascending aorta. Can J Cardiol 2008; 24: 857-859.

6. Cheng $\mathrm{Cl}$, Wu CJ, Hsieh YK, et al. Percutaneous coronary intervention for iatrogenic left main coronary artery dissection. Int J Cardiol 2008; 126: 177-182.

7. 2010 ACCF/AHA/AATS/ACR/ASA/SCA/SCAI/SIR/STS/SVM Guidelines for the Diagnosis and Management of Patients With Thoracic Aortic Disease: Executive Summary. J Am Coll Cardiol 2010; 55: 1509-1544.

8. Woo KM, Schneider JI. High-risk chief complaints I: chest pain the big three. Emerg Med Clin North Am 2009; 27: 685-712.

9. Hagan PG, Nienaber CA, Isselbacher EM, et al. The International Registry of Acute Aortic Dissection (IRAD): new insights into an old disease. JAMA 2000; 283: 897-903.

10. Pratt B, Curci J. Arterial elastic fiber structure. Function and potential roles in acute aortic dissection. J Cardiovasc Surg (Torino) 2010; 51: 647-656. 